\title{
Features of the PHF8/KIAA1718 histone demethylase
}

\author{
Tamaki Suganuma $^{1}$, Jerry L Workman ${ }^{1}$ \\ ${ }^{1}$ Stowers Institute for Medical Research, 1000 E. 50th Street, Kansas City, MO 64110, USA \\ Cell Research (2010) 20:861-862. doi:10.1038/cr.2010.110; published online 20 July 2010
}

Gene regulation mechanisms in cellular events ranging from development to tumorigenesis target the fundamental unit of chromatin structure, the nucleosome, $146 \mathrm{bp}$ of DNA wrapped around a histone octamer. Covalent modifications of histones play key roles in the function of the genome. Histones are methylated on lysine $(\mathrm{K})$ and arginine $(\mathrm{R})$ residues. Methylation has been shown to occur on five $\mathrm{K}$ residues of histone $\mathrm{H} 3$ (K4, K9, K27, K36 and K79), and on four R residues (R2, R8, R17 and R26). H4K20 and H4R3 are also methylated [1,2]. Methylation is a reversible modification regulated by site-specific methyltransferases and demethylases [1]. Demethylases are classified into three groups: peptidylarginine deiminase 4 (PADI 4), lysine-specific demethylase (LSD), and Jumonji C (JmjC)domain-containing proteins. LSD has two members (LSD1 and LSD2), and removes mono-(me) or di-methyl (me2) groups from $\mathrm{H} 3 \mathrm{~K} 4$ or $\mathrm{H} 3 \mathrm{~K} 9$ in an amine oxidase reaction [3]. To date, $98 \mathrm{JmjC}$ domain-containing proteins have been isolated from fission yeast to humans, and classified into seven groups. They catalyze lysine demethylation through an oxidative reaction that requires iron $\mathrm{Fe}(\mathrm{II})$ and $\alpha$-ketoglutarate $(\alpha \mathrm{KG})$ as cofactors [4]. JMJD6 (Jumonji domain containing 6 protein) is iron and 2-oxoglutarate demethylase of ariginine [5]. Demethylase functions have an impact

\footnotetext{
Correspondence: Jerry L Workman

E-mail: JLW@stowers.org
}

on gene regulation through the specific histone substrate preferences of individual demethylases.

The plant homeodomain finger (PHF2/PHF8) proteins (PHF2, PHF8, KIAA1718, spt3, and 4F429) are found in worms, mice and humans, and contain a plant homeodomain (PHD), in addition to a JmjC domain [1]. Importantly, mutations in PHF8 cause inherited X-linked mental retardation (XLMR) and cleft lip/palate [6]. These observations led to questions about how the demethylase activity of PHF8 is regulated, and how PHF8 functions in biological processes that are involved in the development of XLMR.

Recently, Yu et al. demonstrated the catalytic specificity of human PHF8 by determining its crystal structure [7]. Working with wild type (WT) PHF8 and a PHD deletion mutant (c-PHF8) discussed below, they showed that human c-PHF 8 consists of $16 \alpha$ helices and 12 $\beta$-strands, arranged in a similar manner to a known JmjC-domain-containing histone demethylase (JHDM1a) [7]. In the presence of $\mathrm{Fe}^{2+}$ and $\alpha \mathrm{KG}, \mathrm{Fe}^{2+}$ sits in the center of the c-PHF8 catalytic core, which consists of nine hydrophobic residues. The binding of $\alpha \mathrm{KG}$ to $\mathrm{Fe}^{2+}$ does not drastically change the conformation, however it causes the side chain of Y257 to turn towards the substrate binding channel, to be in a position to contact the methyl group of $\mathrm{K} 9 \mathrm{me} 2$. With $\alpha \mathrm{KG}$ and $\mathrm{Fe}^{2+}$, the active center is too narrow to receive the trimethyl group. Recombinant human PHF8 was able to demethylate $\mathrm{H} 3 \mathrm{~K} 9 \mathrm{me} 2$ and
H3K9me1, as measured by mass spectrometry. A comparison of the c-PHF8 and JHDM1a structures suggests that the c-PHF8 and JHDM1a, which have different lengths and low sequence homology, are responsible for the different binding affinities to $\mathrm{H} 3 \mathrm{~K} 36$. JHDM1a binds methyl H3K36, while PHF8 does not. PHD domain adjacent to the catalytic domain in PHF8 may recognize methyl $\mathrm{H} 3 \mathrm{~K} 9$ and contribute to its demethylation. By contrast, the PHD domain of JHDMla which is distal to its catalytic domain may recognize a different methyl lysine (K36).

Yang, et al. demonstrated a role for the PHD by analyzing the crystal structure of Caenorhabditis elegans KIAA1718 (KDM7A) with H3K9me2, $\mathrm{H} 3 \mathrm{~K} 27 \mathrm{me} 2$ and $\mathrm{H} 3 \mathrm{~K} 4 \mathrm{me} 3$ peptides in the presence of $\mathrm{Fe}^{2+}[8,9]$. The PHD domain of ceKIAA1718 interacts with the catalytic core in a manner similar to human PHF8, and the C-terminal coiledcoil region interacts with the catalytic core. The PHD domain of ceKIAA1718 forms a complex with residues 1-6 of the H3K4me3-containing peptide, however, mutations in the binding residues in the PHD maintain the secondary structure of this JmjC enzyme. Thus, substrate binding is not required to maintain its structure. Lin et al. addressed the role of the PHD of ceKIAA1718 in vivo [10]. The PHD deletion mutant expressed in Tn5 cells using a baculovirus system does not show demethylase activity against $\mathrm{H} 3 \mathrm{~K} 9 \mathrm{me} 2$ and $\mathrm{H} 3 \mathrm{~K} 27 \mathrm{me} 2$ [10], which is not consistent with the crystal structure data. Chromatin im- 
munoprecipitation (ChIP)-sequence analysis of ceKIAA1718 in WT and KIAA1718-knockout $C$. elegans suggests that $\mathrm{H} 3 \mathrm{~K} 9 \mathrm{me} 2$ and $\mathrm{H} 3 \mathrm{~K} 27 \mathrm{me} 2$ are reduced at ceKIAA1718-bound promoter regions, whereas $\mathrm{H} 3 \mathrm{~K} 4 \mathrm{me} 3$ is enriched at these promoters in WT animals. Thus, in vivo the ceKIAA1718 PHD domain may recognize $\mathrm{H} 3 \mathrm{~K} 4 \mathrm{me} 3$ but the enzyme demethylates $\mathrm{H} 3 \mathrm{~K} 9 \mathrm{me} 2$ and H3K27me2. However, effects of these activities on gene expression have not been demonstrated.

The biological significance of PHF8 demethylation was studied by Qiu et al., who showed that loss of PHF8 demethylation, for example in XLMR, affects retinoic acid (RA)-induced neuronal differentiation [11]. Interestingly, ChIP data revealed that PHF8 is recruited to target genes of the RA receptor (RAR), where it functions as an RAR coactivator [11]. RAR $\alpha$-driven transcription was reduced in cells expressing a catalytic mutant (H247A) of PHF8 compared to cells expressing WT PHF8. K9me2 demethylation was observed at RAR target genes by ChIP assay, however, whether demethylation of $\mathrm{K} 9 \mathrm{me} 2$ by PHF8 is required for RAR-dependent transcription and differentiation remains unclear. The features of PHF8 interaction with RAR need to be identified. Other studies support the hypothesis that PHF8/PHF2 family members function as transcription cofactors. Huang et al. showed that KIAA1718 (KDM7A) is required for the demethylation of H3K9me 2 and H3K27me2 at the promoter region of the FGF4 gene by ChIP assay, and KIAA1718 is required for FGF4 transcription in mouse embryonic stem cells [12]. Since the expression levels of KIAA1718 are significantly higher than those of PHF8 in neuronal precursor cells [12], KIAA1718 might be responsible for the specific regulation of these $\mathrm{H} 3$ epigenetic markers at the FGF4 gene at this stage. Tissue or stage specific functions may be a reason for the large number of different JmjCdomain-containing demethylases.

Activation of rRNA gene transcription by PHF8 through interaction with RNA polymerase I (Pol I) and a WDR5containing $\mathrm{H} 3 \mathrm{~K} 4$ methyltransferase complex has been demonstrated [13]. Recently, Zhu et al. showed that K9me2 demethylation in the promoter region of the human rDNA gene that results in rRNA transcription requires PHF8 [14]. H3K9me2 occupancy on rDNA gene by ChIP assay in control cells displayed two peaks, while H3K9me2 occupancy was stronger but more diffuse in PHF8 knockdown cells. Thus, these data suggest that PHF8 functions as part of a multienzyme complex upstream of rDNA genes. Moreover, pre-rRNA levels are reduced in the cells over-expressing an F279S mutant of PHF8, which was identified in an XLMR patient.

Reviewing studies on PHF8 family members suggests that comparing features of the complete protein sequences may reveal biological specificity in the functions of these demethylases. In addition, determining the features of substrates will support our understanding of how the catalytic activity of PHF8 proteins is coordinated. Mutations in demethylase genes may be candidates for diagnosis of human disease, however, the real challenge will be how to treat inherited diseases through recovery of demethylase activity.

\section{Acknowledgements}

We thank Dr Chris Tachibana for editing this report. This work was supported by the Stowers Institute for Medical Research.

\section{References}

1 Klose RJ, Kallin EM, Zhang Y. JmjCdomain-containing proteins and histone demethylation. Nat Rev Genet 2006; 7:715-727.

2 Pal S, Sif S. Interplay between chro- matin remodelers and protein arginine methyltransferases. $J$ Cell Physiol 2007; 213:306-315.

3 Shi Y. Histone lysine demethylases: emerging roles in development, physiology and disease. Nat Rev Genet 2007; 8:829-833.

4 Klose RJ, Yan Q, Tothova Z, et al. The retinoblastoma binding protein $\mathrm{RBP} 2$ is an H3K4 demethylase. Cell 2007; 128:889-900.

5 Chang B, Chen Y, Zhao Y, Bruick RK. JMJD6 is a histone arginine demethylase. Science 2007; 318:444-447.

6 Laumonnier F, Holbert S, Ronce N, et al. Mutations in PHF8 are associated with $\mathrm{X}$ linked mental retardation and cleft lip/cleft palate. J Med Genet 2005; 42:780-786.

7 Yu L, Wang Y, Huang S, et al. Structural insights into a novel histone demethylase PHF8. Cell Res 2010; 20:166173.

8 Yang Y, Hu L, Wang P, et al. Structural insights into a dual-specificity histone demethylase ceKDM7A from Caenorhabditis elegans. Cell Res 2010; 20:886-898.

9 Allis CD, Berger SL, Cote J, et al. New nomenclature for chromatin-modifying enzymes. Cell 2007; 131:633-636.

10 Lin H, Wang Y, Wang Y, et al. Coordinated regulation of active and repressive histone methylations by a dual-specificity histone demethylase ceKDM7A from Caenorhabditis elegans. Cell Res 2010; 20:899-907.

11 Qiu J, Shi G, Jia Y, et al. The X-linked mental retardation gene PHF8 is a histone demethylase involved in neuronal differentiation. Cell Res 2010; 20:908918.

12 Huang C, Xiang Y, Wang Y, et al. Dual-specificity histone demethylase KIAA1718 (KDM7A) regulates neural differentiation through FGF4. Cell Res 2010; 20:154-165.

13 Feng W, Yonezawa M, Ye J, Jenuwein T, Grummt I. PHF8 activates transcription of rRNA genes through $\mathrm{H} 3 \mathrm{~K} 4 \mathrm{me} 3$ binding and H3K9me1/2 demethylation. Nat Struct Mol Biol 2010; 17:445450.

14 Zhu Z, Wang Y, Li X, et al. PHF8 is a histone $\mathrm{H} 3 \mathrm{~K} 9 \mathrm{me} 2$ demethylase regulating rRNA synthesis. Cell Res 2010; 20:794-801. 